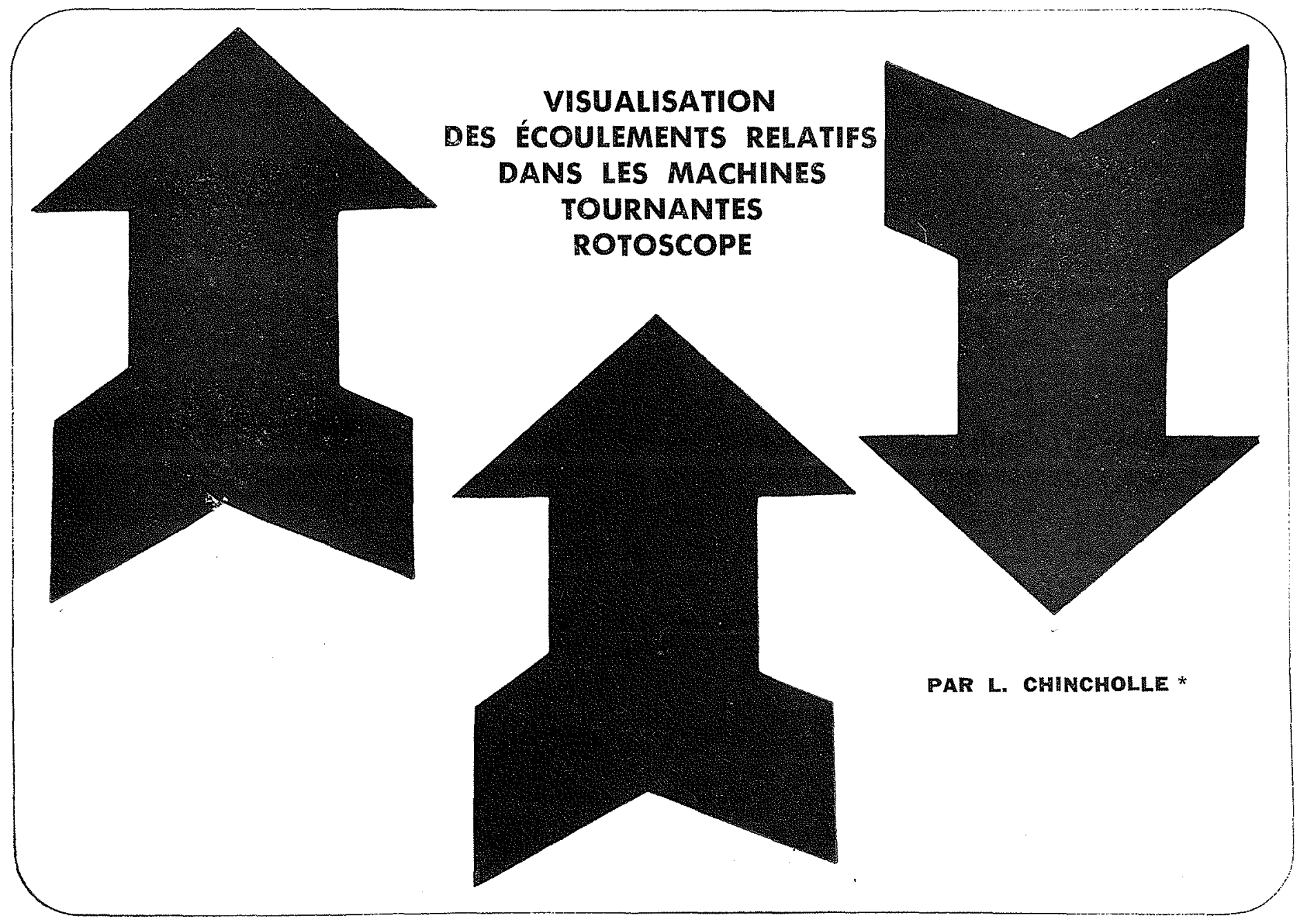

Il est souvent inléressant de connaître les lignes de courant du fluide ou les trajectoires de certaines particules en suspension à l'intérieur d'une machine tournante. Nous rappellerons succinctement les diverses techniques utilisables en insistant particulièrement sur l'emploi du rotoscope. Cet appareil a pour but $d$ ' «arrêter » le mouvement de rotation d'une facon continue et de ne Iaisser apparaitre que le mouvement relatif.

\section{Particularités de la visualisation des écoulements dans les pompes centrifuges Caractère du mouvement.}

Le mouvement absolu d'une particule liquide est la résultante du mouvement relatif du fluide par rapport aux aubes et du mouvement d'entrainement du repère lié aux aubes. Ce dernier est une rotation.

Les caractéristiques de cette rotation sont connues et la vitesse circonférentielle qu'elle entraine facile à calculer. Plus délicate à atteindre est la vitesse relative due à des accélérations complexes. Aussi avons-nous essayé de l'observer directement par une technique de visualisation appropriée.

\section{Matérialisation des lignes de courant}

La technique des filets colorés utilisant un liquide de même densité que l'eau ne peut pas s'appliquer.

\footnotetext{
* Maitre-Assistant à la chaire d'Electrotechnique, Faculté des Sciences de Paris.
}

Les violents tourbillons les effacent très vite et, dans un cycle fermé le liquide se souille rapidement.

La technique des fils disposés sur la roue mobile nous a donné de bons résultats. Elle permet une observation prolongée. Toutefois les fils s'usent et doivent être remplacés. Leur nombre fini empêche de fixer avec précision le centre des multiples tourbillons qui apparaissent.

L'apport de particules brillantes aide également à matérialiser les lignes de courant. Leur densité doit être sensiblement égale à celle du fluide sinon l'effet important de l'accélération leur impose des trajectoires différentes de celles des lignes de courant.

Souvent les expérimentateurs utilisent des bulles d'air pour simuler ces lignes de courant. Nous avons aussi été tentés de le faire. Mais la plus grande prudence est de rigueur dans l'analyse des résultats. La méthode n'est pas à conseiller dans une pompe. Tantôt, sous l'influence de la poussée d'Archimède, les bulles se rassemblent au centre des tourbillons, tantôt, par effet fusée, elles deviennent autonomes et quasiment insensibles au schéma d'écoulement du liquide [2].

L'emploi de substances différentes du fluide étudié réclame toujours une interprétation serrée des résultats.

\section{Différents moyens d'observation.}

L'analyse d'un mouvement peut se faire soit par vision directe soit par l'intermédiaire de la photo graphie. L'un ou l'autre de ces procédés a été choisi suivant les cas. 
Soit l'écoulement de I'eau à l'intérieur d'une pompe centrifuge dont la roue est animée d'un mouvement de rotation uniforme. Sous un éclairage continu, notre ceil ne relève aucun renseignement intéressant à cause de la persistance des impressions lumineuses. Une photo instantance indiquera la répartition de fines particules étrangères mais sans donner ni la direction ni l'intensité de la vitesse. Avec la technique des fils le sens de l'écoulement apparait.

Si nous réalisons une pholo avec un temps de pose déterminé, la vitesse absolue sera définie en grandeur et en direction mais pas toujours en sens. Certains expérimentateurs en déduisent la vitesse relative compte tenu de la vitesse d'entrainement. Cette technique nous apparait délicate mais, bien appliquée, elle foumit de bons résultats numériques. Le tracé des lignes de courant relatives découle alors de ces mesures. Nous lui préférons l'observation directe du mouvement relatif, à l'œil nu ou par photographie.

Deux appareils permettent de l'atteindre: le stroboscope et le rotoscope. Nous rappellerons d'abord le fonctionnement du premier car sa connaissance aide à mieux comprendre l'utilisation du second.

Avant d'aborder cette étude, nous signalerons l'emploi de l'appareil photographique monté sur un axe qui tourne à la vitesse de rotation de l'objet. Avec des temps de pose suffisants les trajectoires relatives s'inscrivent. Nous reprochons à cette méthode une mise en wuvre très délicate el surtout l'impossibilité de l'observation visuelle directe.

\section{Rotoscope}

La stroboscopie utilise un générateur d'éclairs très brefs se succédant à intervalles réguliers. Quand on éclaire une roue en rotation uniforme, celle-ci parait arètée si la fréquence des éclairs égale sa vitesse de rotation. Seul subsiste le mouvement relatif que l'emploi de fils, par exemple, met en évidence. Bien que l'objet ne soit éclairé qu'une fois par tour, la persistance des impressions lumineuses sur l'œil assure la continuité du mouvement.

Le stroboscope permet une observation visuelle directe facile. Des artifices d'éclairement peuvent améliorer la méthode. Ainsi, avec une fréquence. d'éclairs quadruple de celle d'une roue en croix de St-André, les vues de tous les quadrants constitués par les aubes symétriques sont rassemblées en une seule. Les lignes de courant simulées par les fils sont plus denses et plus apparentes. Elles traduisent mieux l'écoulement réel et permettent aussi de vérifier la similitude des écoulements dans les quadrants. Une photo dont le temps de pose couvre plusieurs éclairs accentue encore cet effet.

L'application de cette technique au cas de particules en suspension dans le liquide ne donne pas de résultats intéressants. Soit une fréquence d'éclairs égale à celle de la roue. Quand le temps de pose est inférieur à celui qui sépare deux éclairs on obtient un cliché instantané identique à celui que nous avons déjà rencontré. S'il couvre plusieurs éclairs un nombre égal de vues se superposeront

sur une même photo. Les positions d'une particule à des instants voisins, séparés par une période, définissent théoriquement sa trajectoire relative en pointillés. Mais la multiplicité des bulles et des points relativement éloignés l'un de l'autre rend impossible tout tracé. Les photographies sont inexploitables.

D'autre part, le stroboscope ne permet pas de suivre les fluctuations des lignes de courant durant un tour entier. Les informations qu'il donne sont incomplètes et discontinues. L'histoire de la particule échappe à l'observateur.

'Toutefois celle technique n'est pas rejeter. D'un emploi très facile, avec des fils par exemple, elle permet d'observer à loisir l'écoulement relatif permanent, la forme des lignes de courant, l'emplacement des tourbillons, la stabilité de l'ensemble et son évolution lorsque certains paramètres varient.

\section{Rotoscope.}

Les inconvénients du stroboscope, dus essentiellement à la discontinuité de l'observation, ne se rencontrent plus dans la technique du rotoscope. Nous avons construit cet appareil en nous inspirant d'une idée de Thoma [1]. Sa mise au point a été assez délicate.

Principe de l'appareil. La figure 1 a représente le miroir classique de Poggendorff. Quand il tourne d'une angle $\alpha$ autour du point $O$, l'image $\mathrm{P}^{\prime}$ de $\mathrm{P}$ tourne d'un angle $2 \alpha$. Il est évident que si l'objet tourne d'un angle $2 \alpha$ et le miroir d'un angle $\alpha$, l'image $P^{\prime}$ reste fixe. L'image et l'objet sont symétriques par rapport à un plan perpendiculaire au miroir.

Notre but était d'appliquer cette propriété pour observer le système en rotation constitué par la pompe. Celle-ci tournant à la vitesse $2 \omega$, il suffisait de prévoir une rotation $\omega$ du miroir pour que l'image soit fixe. Mais il fallait, ce qui est irréalisable, que le miroir soit dans la pompe.

L'artifice du prisme de Wollaston (fig. 1 b) pallie cet inconvénient. La face ABCD constitue le miroir et les faces non parallèles réalisent la translation du point O vers l'avant. Un rayon lumineux issu

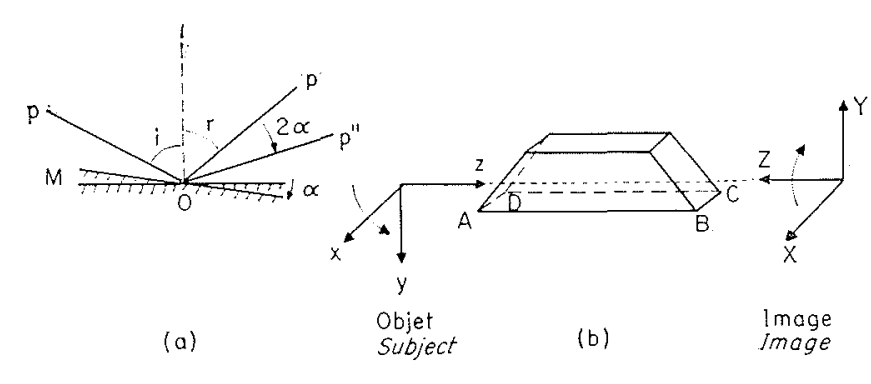

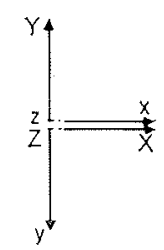

(c)

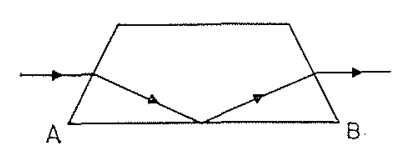

(d) 
de l'objet subit successivement une réfraction, une réflexion et une nouvelle réfraction (fig. $1 d$ ).

Comme le schématise la figure $1 b$, le prisme domne d'un trièdre direct un autre trièdre direct mais déphasé par rapport à l'objet. Cette particularité apparaît sur la figure $1 c$ qui est une vue de gauche de la figure $1 b$. On roit que le prisme introduit une symétrie par rapport à un plan. $\vec{Z}$ et $\vec{z}$ représentent les vecteurs rotation. Le problème est ramené à celui d'un simple miroir.

Théoriquement, nous pouvons donc «arrêter» le mouvement de rotation. L'œil percoit le mouvement relatif d'une facon continue. La persistance des impressions lumineuses n'a plus à intervenir.

L'observation à travers le prisme présente quelques particularités que nous résumons brièvement (photo 1):

- le sens de rotation de la pompe est inversé;

- si le miroir est fixe et si la roue tourne à la vitesse $\vec{\omega}$, l'image tourne à la vitesse $-\vec{\omega}$;

- si la roue est fixe et si le miroir tourne à la vitesse $\vec{\omega} / 2$ l'image tourne à la vitesse $-\vec{\omega}$;

- si la roue tourne a la vitesse $\vec{\omega}$ et le miroir à la vitesse $\vec{\omega} / 2$; l'image est fixe. Il suffit, pour le montrer, de superposer les deux mouvements précédents;

- plus généralement, si $\vec{m}$ et $\vec{n}$ sont respectivement les vitesses de rotation de la roue et du miroir, la vitesse de l'image sera :

$$
\vec{\omega}=-\vec{m}+2 \vec{n}
$$

Le rotoscope comprend essentiellement un prisme de Wollaston et son dispositif d'entraînement à une vitesse de rotation rigoureusement moitié de celle de la roue.

Le prisme présente une base trapézoïdale dont les còtés non parallèles forment un angle de $45^{\circ}$ avec la grande base. Sa section droite est un carré de $30 \mathrm{~mm}$ de cotci environ (fig. 2 a) (photo 2).

Il est placé dans un cylindre support $\mathrm{S}$. Des vis

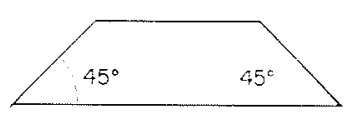

(a)

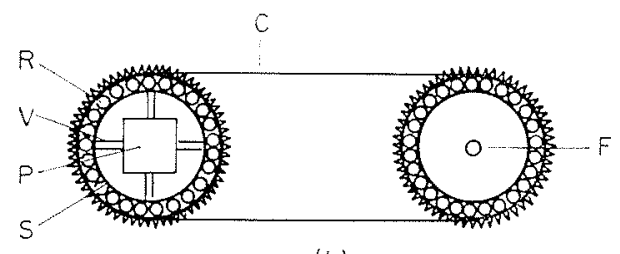

(b)

2/ C : Chaine de transmission / Chain drive;

F : Entraînement / Drive shaft;

P : Prisme / Prism;

$\mathrm{R}$ : Roulement à billes / Ball bearing;

S : Cylindre support / Carrier cylinder

$\mathrm{V}: \mathrm{Vis} /$ Screw; $\checkmark$ permettent d'ajuster sa position et de le fixer solidement. La figure $2 b$ représente le montage du prisme et son entraînement. Le support $\mathrm{S}$ est solidaire d'un pignon sur lequel engrène une chaine (photo 3). Un montage analogue du côté menant est mis en mouvement par le dispositif moteur.

Après des essais infructueux avec plusieurs transmissions flexibles, nous avons porté notre choix sur une transmission par arbre électrique. L'une des machines est placée en bout d'arbre de la pompe par l'intermédiaire d'un pignon réducteur dans le rapport $1 / 2$. L'autre entraine le rotoscope. Le synchronisme est ainsi assuré. Nous avions demandé un groupe électrique réalisant lui-même le rapport moitié des vitesses mais le constructeur n'a pas pu nous le fournir. Les ennuis qui se présentent proviennent en général d'oscillations qui s'amorcent lorsque la fréquence de résonance des appareils est roisine de la vitesse de rotation. Le synchronisme existe en moyenne mais pas a chaque instant.

La mise en place du prisme dans son logement soulève des difficultés. L'axe optique du prisme doit cöncider avec l'axe de rotation. Après de nombreux essais, correspondant à des méthodes de réglage différentes, nous avons obtenu des résultats acceptables. De la perfection du réglage dépend la stabilité de l'image.

\section{Utilisation de l'appareil.}

La vitesse du moteur d'entrainement de la pompe varie à volonté depuis zéro jusqu'à plusieurs milliers de tours par minute. Le rotoscope, suivant fidèlement le mouvement de la roue, en donne une image fixe. Certes, quelques oscillations se produisent encore mais elles ne gênent guère l'observation du mouvement relatif de l'écoulement. Celui-ci apparait clairement.

Le rotoscope donne une vue nouvelle des phénomènes et facilite beaucoup leur compréhension, notamment en permettant de suivre leur évolulion en fonction de la vitesse de rotation.

Afin d'atteindre avec plus de précision les mouvements rapides il faut avoir recours à la photographie. A cause de la persistance des impressions lumineuses, l'œil tend à faire la moyenne des phénomènes. Le choix du temps de pose présente une grande importance.

Nous disposons d'un appareil photographique pouvant utiliser des films du type Polaroïd dont nous sommes satisfaits. Nous avons été amenés à lui adapter un téléobjectif. Le temps de pose minimal est alors de $1 / 100^{\circ}$ de seconde. Pour certaines vues, relatives à la vérification de l'effet fusće, il a fallu envisager des temps de pose de lordre de $50 \mu \mathrm{s}$. Certains observateurs ont photographic ce phénomène avec un temps de pose d'une milliseconde par exemple. Ils en ont déduit des vitesses en rapport avec ce temps alors que la trajectoire caractéristique de l'effet fusée était décrite en 50 us. La valeur de la vitesse qu'ils ont calculée doit donc ètre multipliée par le coefficient 20 .

Pour atteindre des temps de pose d'une valeur aussi faible nous avons utilisé l'éclair produit par un flash construit spécialement. Par un jeu de transformateurs, de condensateurs et de selfs, on peut régler la durée efficace de l'éclair.

La figure 3 montre que suivant les valeurs choisies la durée d'exposition d'une plaque sensible 
3/

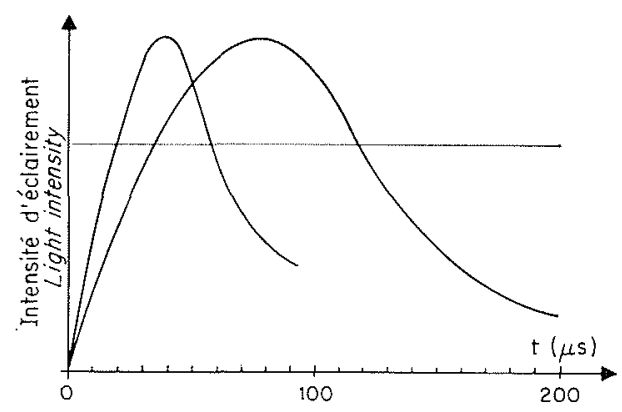

varie considérablement. Elle peut passer de quelques microsecondes à des valeurs mille fois plus grandes.

D'autre part, la forme de la courbe présente une dissymétrie qui nous sert à voir le sens de la trajectoire. La partie floue de celle-ci, provenant de la «queue» de l'éclair, caractérise l'instant final.

La sensibilité de la plaque photographique étant fixée, il faut déterminer le temps de pose. Pour cela nous utilisons une turbine à palier à air sur laquelle est peint un point blanc. Sa vitesse réglable est donnée par un compteur électronique. La mesure de la longueur de la trajectoire du repère peruncl de calculer la durée d'exposition et d'étalonner l'appareil.

L'emploi du rotoscope réclame une certaine habitude de la part de l'expérimentateur. Tout ce qui est au repos dans la pompe tourne à la vitesse $-\vec{\omega}(\vec{\omega}$ : vitesse de rotation de la roue). Tout ce qui est en rotation $\vec{\omega}$ se trouve au repos. Par conséquent, tous les organes fixes de l'installation troubleront la visibilité. Par exemple, Je tuyau d'entrée au centre de la pompe cache partiellement un quadrant. A l'oeil nu cel organe crée un flou qu'on distingue à peine. En photographie, avec an temps de pose égal aux trois quarts de la période de rotation on obtient la vue nette d'un quadrant seulement. Pour la même raison la transparence du flasque avant devra également etre assurée le mieux possible.

Quand le vecteur rotation d'une particule a pour valeur $\vec{\omega} \varepsilon$ et celui du rotoscope $\vec{\omega} / 2$, la particule paraît tourner à la vitesse $+\vec{\varepsilon}$. La longueur d'une trajectoire relative est donc respectée.

L'image transmise par le rotoscope étant symétrique de l'objet, l'observateur la voit à l'envers. On peut éviter cet ennui en intercalant un miroir dans le trajet des rayons lumineux. Ceci permet également de disposer l'appareil daus une direction quelconque et de faciliter le travail de l'expérincentateur. Il est possible en outre, lors du tirage des photos, de retourner le négatif.

L'utilisation jumelée du roloscope, du générateur d'éclairs et de l'appareil photographique présente l'avantage de découper la trajectoire relative suivant une base de temps quelconque et réglable. L’inconvénient signalé pour la stroboscopie n'intervient pas ici car la fréquence des éclairs peut ètre augmentée à volonté jusqu'à ce que la trajectoire apparaisse. L'accélération du mouvement se déduit directement de la photo. Etant donné un écoulement dans une pompe centrifuge on pent donc connaitre parfaitement le mouvement relatif d'une particule puisqu'on dispose de la trajectoire, de la vitesse et de l'accélération.

Avec une mise au point soignée, le rotoscope présente des avantages indiscutables. Son emploi est agréable, facile et rapide. C'est l'oulil inaispensable aux chercheurs et aux techniciens des plates-formes d'essais qui désirent connaitre les éconlements relatifs dans les machines tournanles hydrauliques el aérauliques.

\section{Résultats expérimentaux}

Nous présenterons quelques photographies relalives à la visualisation des écoulements dans les pompes centrifuges. La comparaison des diverses techniques permettra, tout en faisant apparaître leurs qualités respectives, de se familiariser avec leur interprétation.

\section{Observations générales.}

Les paramètres importants sont:

$\mathrm{N}(\mathrm{tr} / \mathrm{mn})$ : vitesse de rotation de la roue;

$\mathrm{Q}_{v}\left(\mathrm{~m}^{3} / \mathrm{s}\right):$ débil;

$t(\mathrm{~s}):$ lemps de pose.

Nous n'indiquerons pas la valeur d'ouveriure du diaphragme. Etant donnés le temps de pose trìs court ef la faible intensité lumineuse des bulles, il est en général grand ouvert $(4,5)$.

Les films polaroïd employés ont une sensibilité de 3000 ASA.

Photo $n^{\circ}$ \&: Roue A (Croix de Saint-André). Le temps de pose est fixé par la durée de l'éclair du stroboscope. L'étalonnage réalisé à l'aide d'une turbine a palier à air nous a permis de l'évaluer à $15 \mu$ s environ. Nous obtenons ainsi un inslantanć. Mème avec des vitesses de rotation de l'ordre de $2000 \mathrm{tr} / \mathrm{mn}$, la vue d'un point de la rone est nette.

La photo $n^{\circ} 4$ correspond à une vilesse de $600 \mathrm{tr} / \mathrm{mn}$. Les bulles sont très apparentes. On peut déterminer leur diamètre qui est de l'ordre du millimètre. Lorsque la vitesse de rotation augmente, les dimensions des bulles diminuent considézablement.

Photo $n^{\circ} 5: \mathrm{N}=200 ; t=1 / 50 \mathrm{~s}$.

Le mouvement absolu d'une bulle apparail nettement. Le flou correspond à la pale droile de la roue $A$.

Photo $n^{\circ} 6$ : Visualisation par fils. Cne vue instantanée indique la forme de l'écoulement a ce moment-là. Les fils doivent être choisis peu rigides et très courts afin d'éviter qu'ils domnent une valeur moyenne des lignes de courant.

Photo $n^{\circ}$ 7: Visualisation au rotoseope. Cet appareil permet de prendre une pose du mouvement relatif. Ainsi la vue précédente instantanée très nette se transforme en une photo flone avec $t=1 / 100 \mathrm{~s}$. On peut ainsi apprécier les oscillations des lignes de courant.

La partie sombre correspond à l'ombre projetée du canal d'arrivée au centre de la pompe. Vue au rotoscope cette partie fixe semble tourner.

Photo $n n^{\circ} 8$ : Les trajectoires relatives des bulles apparaissent. La photo a été prise à travers le rotoscope.

Photo $n^{\circ} 9$ : Cette photo réunit la visualisation 


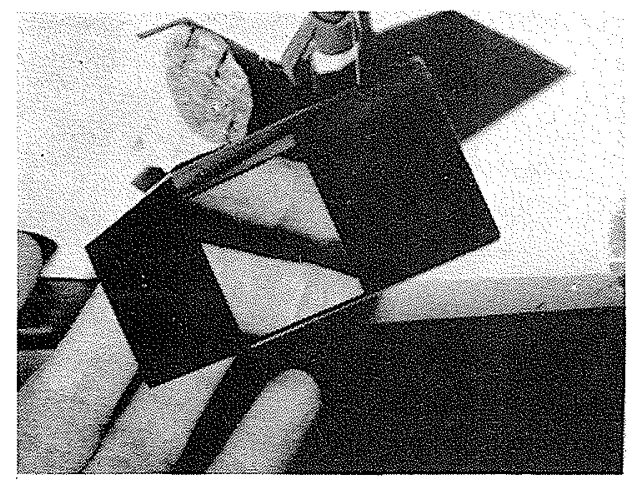

Photo $\mathrm{n}^{\circ} 1$

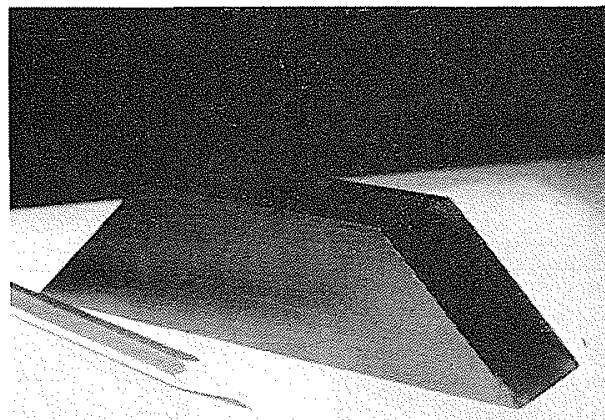

Photo $n^{\circ} 2$

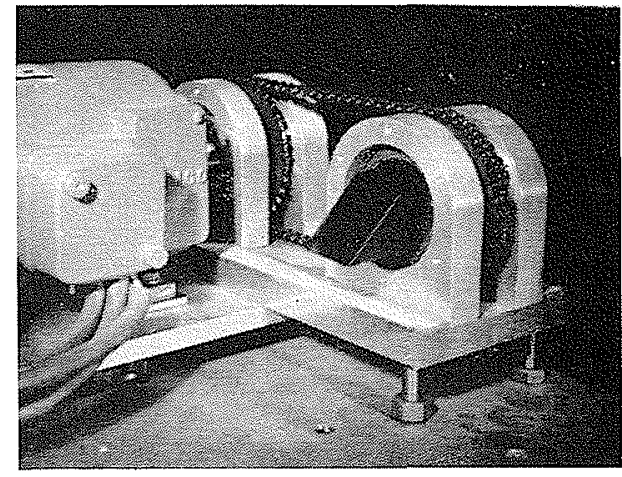

Photo $n^{\circ} 3$

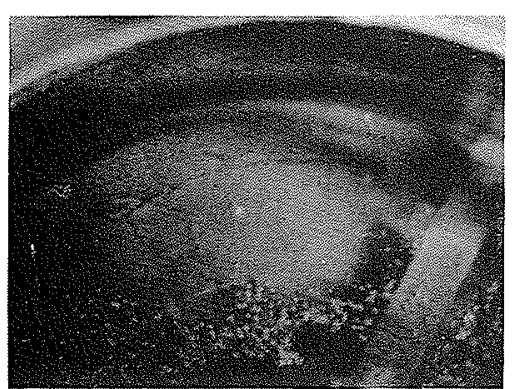

Photo $n^{\circ} 4$

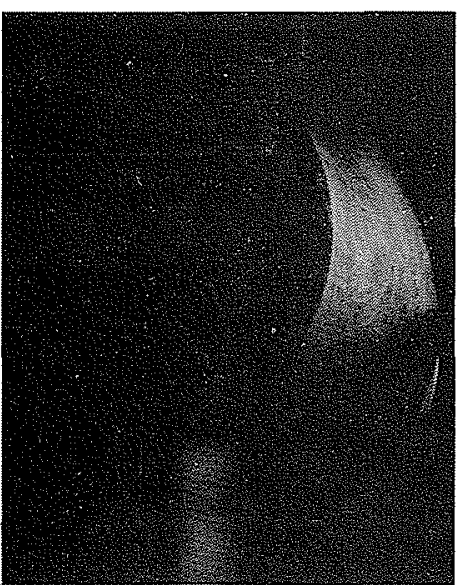

Photo $n^{\circ} 8$

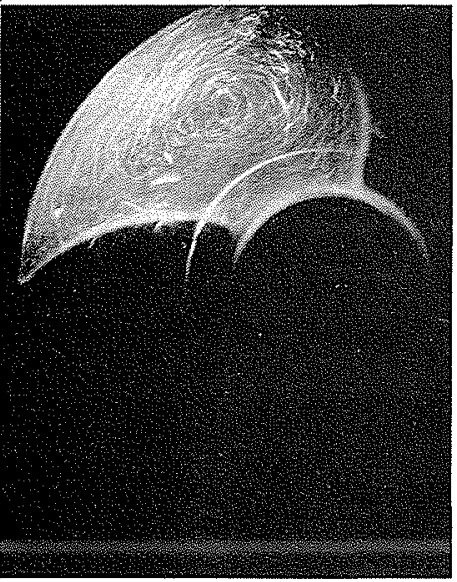

Photo $\mathrm{n}^{\circ} 12$

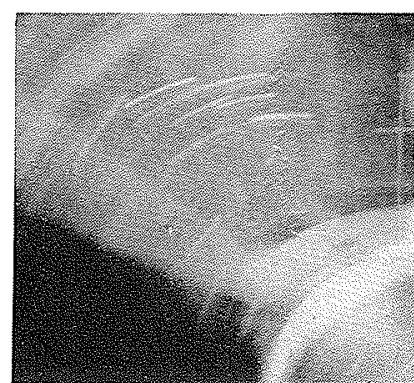

Photo $n^{\circ} 5$

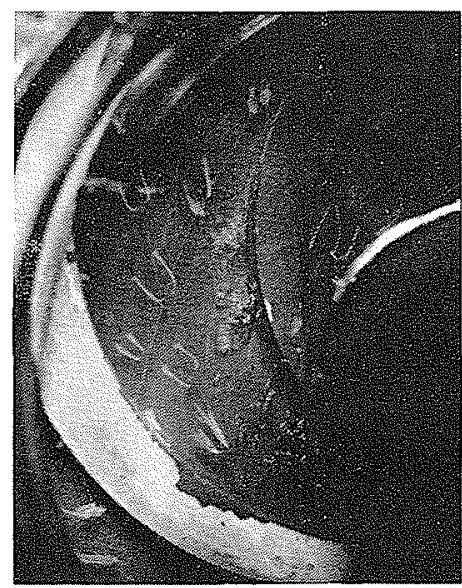

Photo $\mathrm{n}^{\circ} \mathrm{g}$

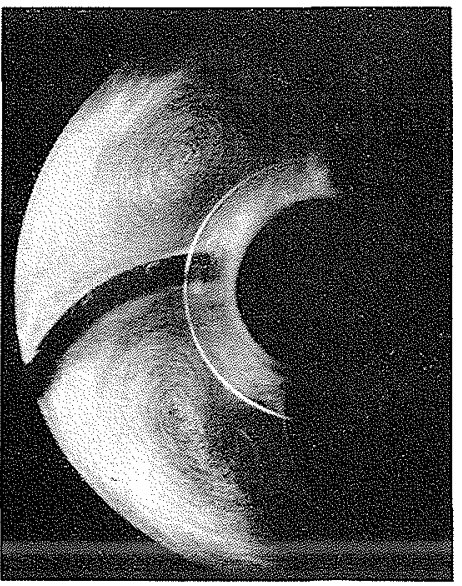

Photo $n^{\circ} 13$

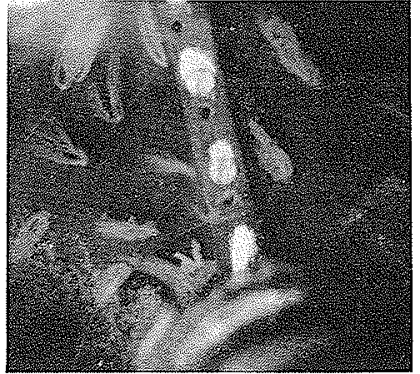

Photo $n^{\circ} 6$

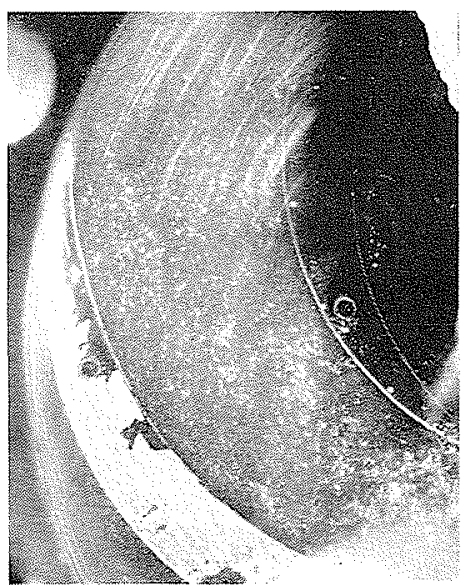

Photo $\mathrm{n}^{0} 10$

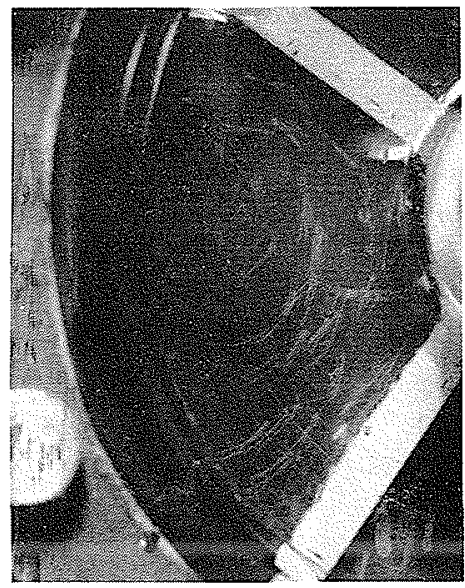

Photo $n^{\circ} 14$

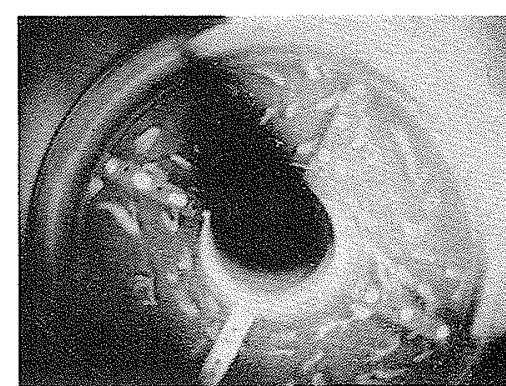

Photo $n^{0} 7$

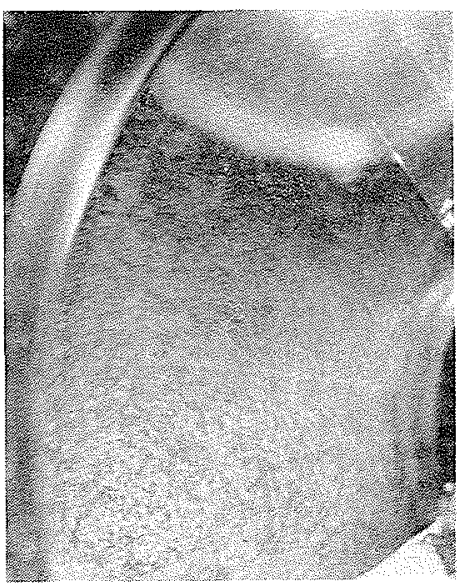

Photo $n^{\circ} 11$

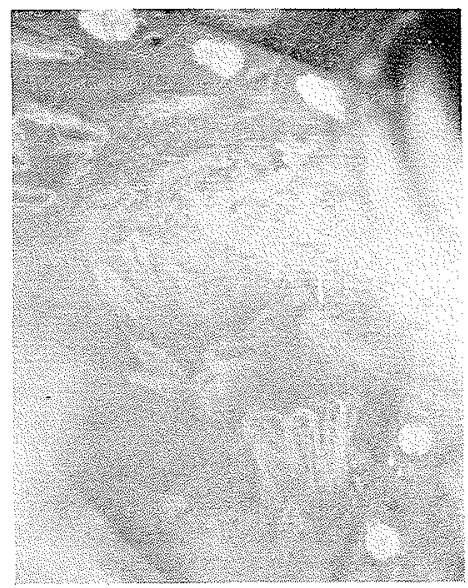

Photo $\mathrm{n}^{\mathrm{o}} 15$ 


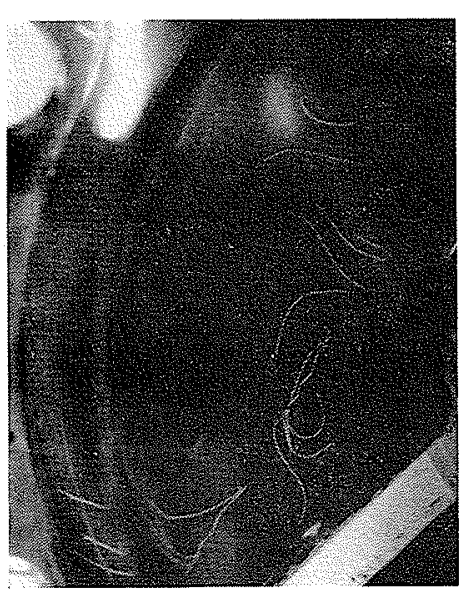

Photo $n^{\circ} 16$

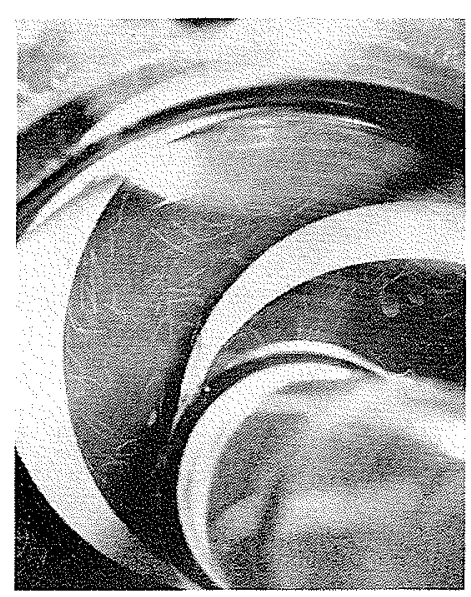

Photo $n^{\circ} 20$

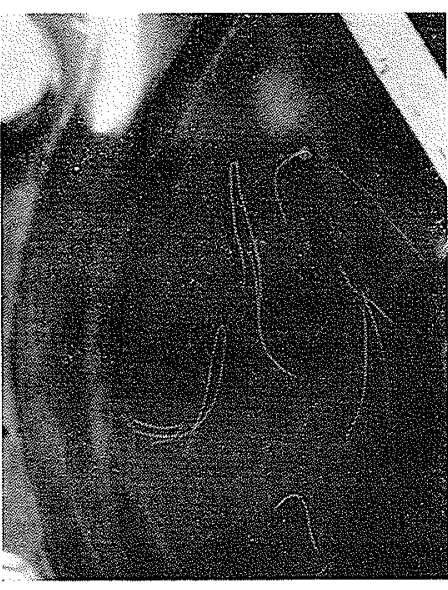

Photo $n^{\circ} 17$

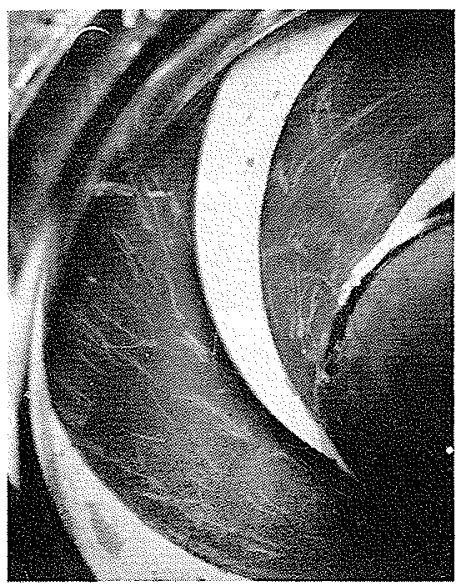

Photo $n^{\circ} 21$

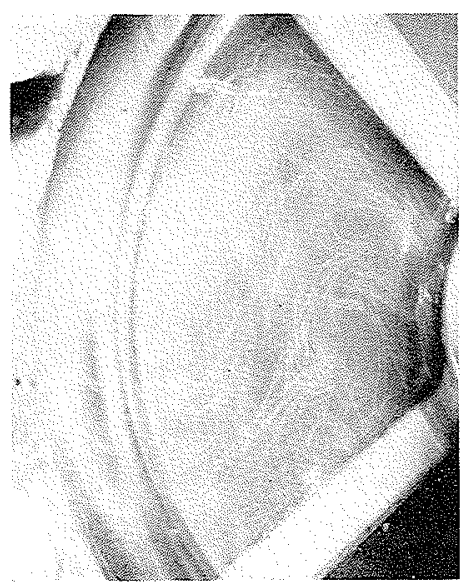

Photo $\mathrm{n}^{\circ} 18$

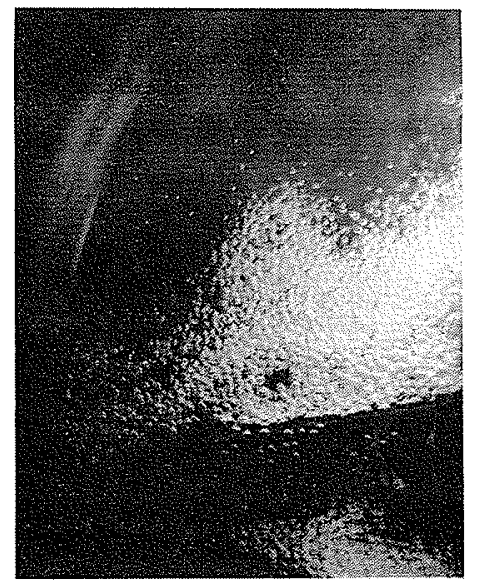

Photo $\mathrm{n}^{\circ} 22$

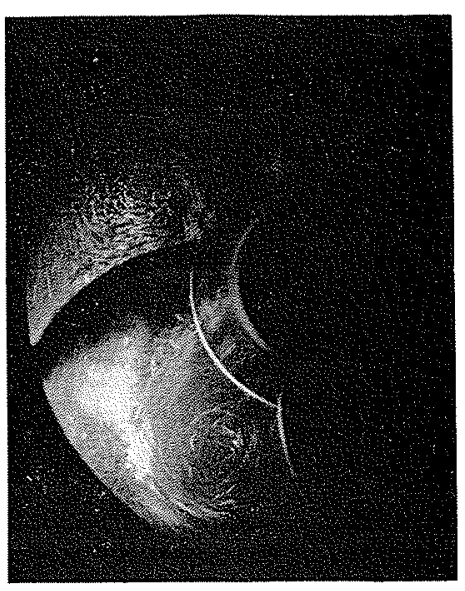

Photo $\mathrm{n}^{\circ} 19$

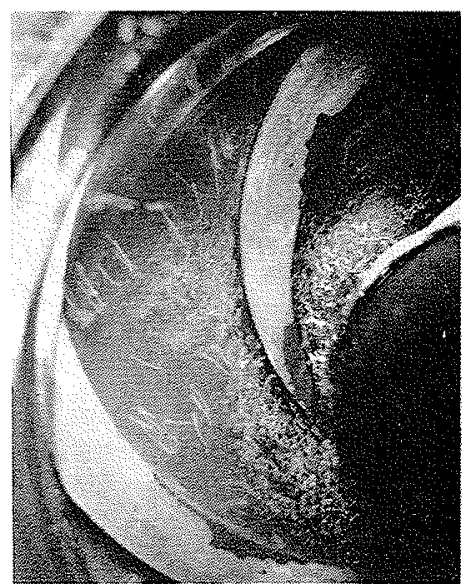

Photo $n^{\circ} 23$

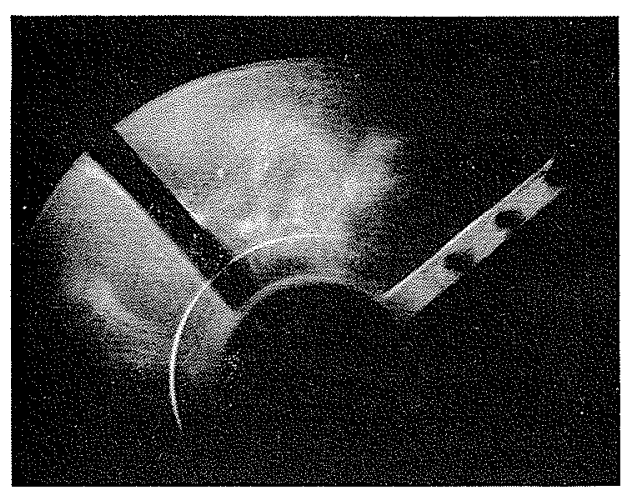

Photo $n^{\circ} 24$

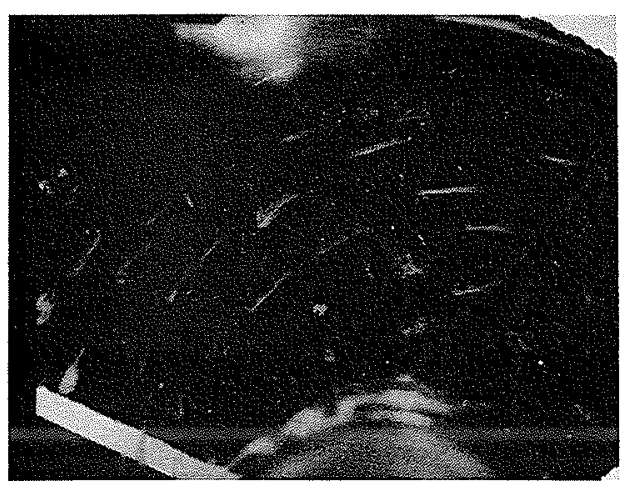

Photo $\mathrm{n}^{0} 27$

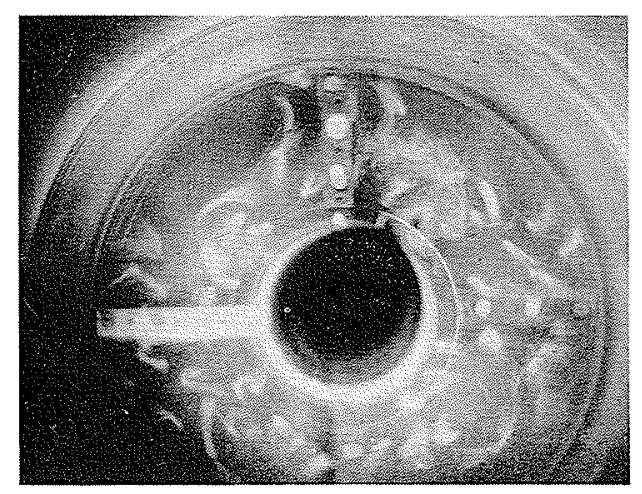

Photo $\mathrm{n}^{\circ} 25$

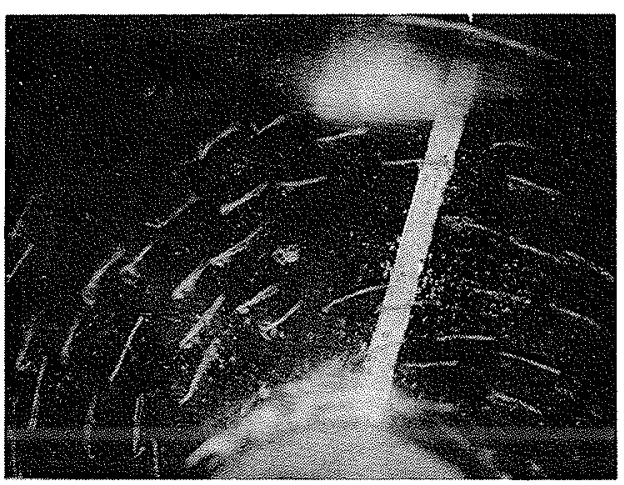

Photo $n^{\circ} 28$

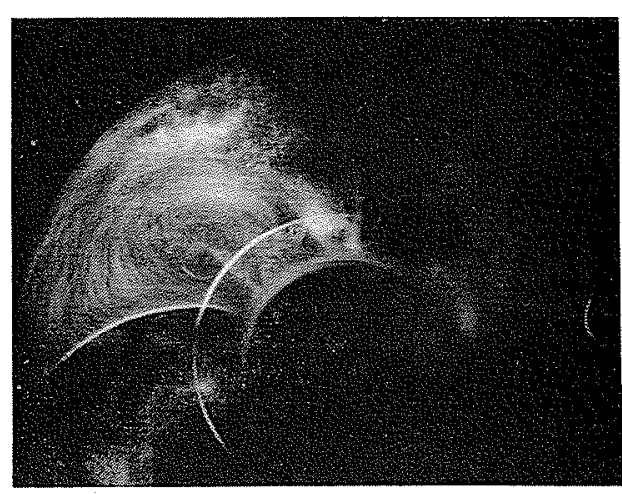

Photo $n^{\circ} 26$

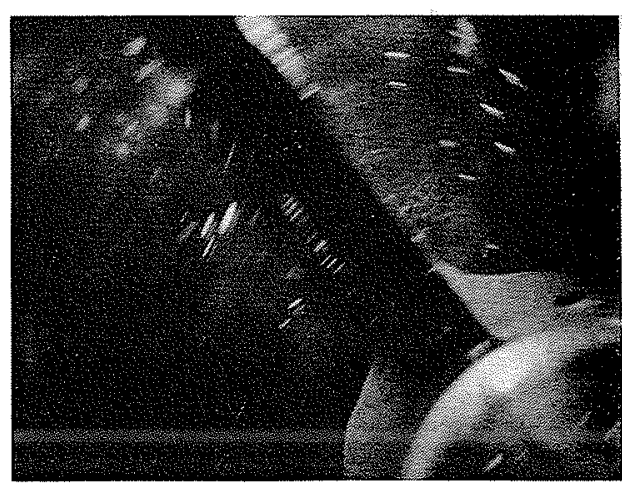

Photo $n^{\circ} 29$ 
par fils et par bulles. Celles-ci se concentrent dans les régions de basse pression $\mathrm{N}=450$.

Photo $n^{\circ} 10$ : Deux éclairages sont superposés: un éclairage continu sur le côté el un éclair en face; $t=1 / 400 \mathrm{~s}$.

Ces clichés montrent les nombreuses possibilités qu'apporte la photographie dans l'observation des bulles à l'intérieur d'une pompe centrifuge.

\section{Différentes formes d'écoulements.}

Nous avons appliqué ces techniques photographiques à la visualisation de certains écoulement particuliers. La roue en forme de Croix de Saint-André a un profil très mauvais. Néanmoins nous l'arons retenue car elle accentue certains phénomènes et elle permet de les comprendre. En transposant ensuite sur des roues mieux profilées, l'œil, déjà habitué à ces perturbations, les décèle et les analyse plus facilement. Le sens de rotation de la roue est toujours le sens direct.

\section{a) Tourbillon de Stodola.}

Ce tourbillon dû à l'inertie du liquide gêne l'écoulement radial. Son centre se déplace suivant le débit.

Photo $n^{\circ} 11$ : Nous avons vouhu meltre en évidence le tourbillon inverse. Pour cela, nous arons arrêté brusquement une pompe en rotation alors qu'elle véhiculait une émulsion. Aussitôt l'arrêt de la roue obtenu, tandis que le liquide était encore en mouvement, nous avons pris la photo. Dans les conditions expérimentales, les bulles matérialisent à peu près les lignes de courant. Leurs diamètres vont en décroissant à partir du centre. Seules les plus petites peuvent se maintenir assez loin malgré la poussée d'Archimède consécutive au champ de pression centrifuge.

Lors du démarrage de la pompe, il est évident qu'un tourbillon analogue apparait. Son sens de rotation est inversé : il est opposé au sens de rotation de la roue.

Photo $\mathbf{n}^{\circ}$ 12: Ce tourbillon apparaît également dans une roue différente. Nous avons utilisé ici le rotoscope $(t=1 / 100 \mathrm{~s}, \mathrm{~N}=1000)$ et un débit nul. La plupart des trajectoires sont celles de graines de pavot peintes en blanc. Il semble que le centre du tourbillon s'étire le long d'une circonférence suivant la profondeur de la roue.

Photo $n^{\circ} 13$ : Elle est identique à la précédente. Les bulles d'air remplacent les graines.

Photo $n^{\circ}$ 1.4: Visualisation par fils. Elle présente l'avantage d'indiquer le sens de rotation du tourbillon. Il est opposé au sens de rotation de la roue (Sans débit).

Photo $n^{\circ} 15$ : Visualisation par bulles et par fils. Les bulles se rassemblent au centre de courbure des lignes de courant. Au fur et à mesure qu'on s'en éloigne leur diamètre se réduit. Ici apparaît la difficulté d'interprétation d'une visualisation par bulles uniquement.

Photos $n^{\text {os }}$ 16-17-18: La première situe le tourbillon (en bas à gauche), la seconde le centre de courbure des lignes de courant (à droite, à l'emplacement du tortillon de fil). Enfin la dernière donne une vue d'ensemble.

Sur la figure 4 , nous avons relevé d'après toutes les vues, de nombreuses lignes de courant que schématise la figure 5 .

Photo $n^{\circ} 19$ : Elle est analogue à la photo $n^{\circ} 12$ mais elle correspond à un débit de $4 \mathrm{~m}^{3} / \mathrm{h}$. Lorsque le débit augmente le centre du tourbillon se déplace dans le sens inverse de la rotation. Entre le tourbillon et l'intrados de l'aube apparait un canal par où s'évacue le liquide.

Le tourbillon obstrue la majeure partie de la section de sortie. On a intérêt à réduire son influence en diminuant son rayon, d'où la forme des aubes en spirale logarithmique notamment.

Photo $n^{\circ} 20$ : L'importance du tourbillon a diminué. Il se scinde en plusieurs tourbillons élémentaires englobés par un écoulement longeant les aubes. La photo a été prise sans débit.

Photo $n^{\circ} 21$ : Avec débit, dans la même roue les tourbillons disparaissent. Seul, un œil exercé parvient à en retrouver quelques traces.

En matérialisant les lignes de courant, la technique de visualisation par fils renseigne sur les améliorations à apporter au profil de la roue.

\section{b) Ecoulement d'une émulsion.}

Si les petites bulles sont entrainées par le liquide, les grosses tendent à gagner les régions de basse pression.

Photo $n^{\circ} 22$ : Avec un débit réduit, les bulles se concentrent vers l'arrière de l'aube ainsi que dans la partie avant située vers le centre de la pompe. On pourrait ainsi tracer des isobares.

Photo $n^{\circ} 23$ : Avec débit. Les régions de basse pression sont mises en évidence. Seules les petiles bulles sont entraînées.

Photos $n^{\text {os } 24-25}$ : Vues au rotoscope $t=1 / 100 \mathrm{~s}$. Eclairage de côté pour la première et de face pour la seconde. Les trajectoires des bulles sont intéressantes à observer mais leur interprétation est souvent délicate $\left(q_{v}=4 \mathrm{~m}^{3} / \mathrm{h}\right)$.

Photo $n^{\circ} 26$ : Elle est identique à la précédente

$4 /$
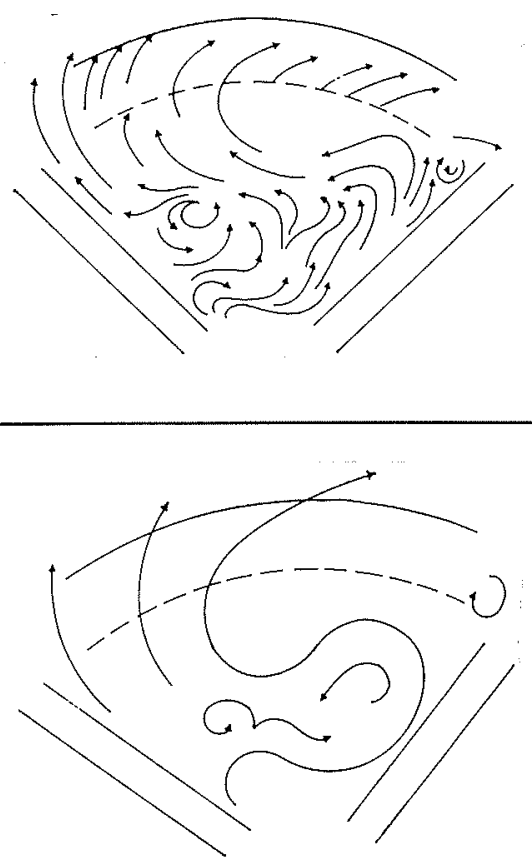

5/ 


\title{
L. CHINCHOLLE
}

mais les aubes sont légèrement profilées (éclairage de còté, $q_{v}=4 \mathrm{~m}^{3} / \mathrm{h}$ ).

On note une différence importante dans l'allure des lignes de courant suivant la forme des aubes sur les photos nos 24 et 26.

c) Roue sans aubes. Couche limite.

Cette roue sans aubes a élé réalisée avec deux joules distantes de $3 \mathrm{~mm}$. L'entraînement de l'eau se fait uniquement par viscosité. Les lignes de courant sont des spirales logarithmiques. Elles coupent les rayons sous un angle de $65^{\circ}$ environ (photo $\mathrm{n}^{\circ} 27$ ).

La photo $\mathrm{n}^{\circ} 28$ représente un écoulement avec émulsion. La vitesse de rotation est de $100 \mathrm{tr} / \mathrm{mn}$.

Sur ces vues nous rencontrons des tortillons de fils. Ils signalent des écoulements anormaux : tourbillons ou écoulements de retour [3].

L'observation au rotoscope (visuelle ou photographique) renseigne sur la stabilité des lignes de courant.

L'étude d'un tel écoulement n'a pas encore été faite. Elle pourrait apporter des renseignements sur la nature de la couche limite existant le long des joues latérales et le cas échéant sur un obstacle.

La photo $\mathbf{n}^{\circ} 29$ montre que la couche limite s'épaissit dans certaines régions. Ceci se traduit par la stabilité des bulles dont la trajectoire absolue devient une circonférence. La roue tourne dans le sens direct. On relève deux de ces zones caractéristiques : en bout de pale à l'arrière et en début de pale à l'avant.

Conclusion

Nous avons présenté quelques exemples de visualisation des lignes de courant et des trajectoires de corpuscules à l'intérieur d'une pompe centrifuge. Il est possible d'utiliser le rotoscope dans l'étude des écoulements de fuites qui se produisent le long des pales ou à leur extrémité et dans l'étude de la cavitation. Les zones perturbées sont ainsi accessibles à l'observation visuelle et à la photographie.

Associé à la visualisation par fils ou par corpuscules, le rotoscope apparait comme l'outil indispensable aux chercheurs et aux techniciens des platesformes d'essais qui désirent connaître les écoulements relatifs dans les machines tournantes hydrauliques et aérauliques.

Bibliographie

[1] Thom (D.). - Die Neue Wasserturbinenversuchsanstalt von Briegleb, Hansen und Co. in Gotha. Gotha Engelhard, Rhyersche Hofbuchdruckerei, 1928.

[2] Chincholle (L.). - Etude de l'écoulement d'une émulsion. Thèse Université de Paris, 1967. La Honille Blanche, $\mathrm{n}^{\circ} 5-1967$ et $\mathrm{n}^{\circ} 2-1968$.

[3] Peube (J.-L.) et Kreith (F.). - Ecoulement permanent d'un fluide visqueux incompressible entre deux disques parallèles en rotation. Journal de mécanique, vol. $5, \mathrm{n}^{\circ} 2$ (juin 1966 ).

\section{Abstract}

The visual observation of relative flow in rotating machines. The rotoscope

\author{
by L. Chincholle *
}

It is often useful to be able to establish the fluid stream lines or the paths of given particles in suspension in a rotary machine. The various methods whereby this can be achieved are reviewed in this article, with special emphasis on the use of the rotoscope, an instrument designed to 'freeze' the rotational motion and only leave relative motion visible.

Where stream lines are made visible by the addition of different substances to the fluid under investigationespecially gas bubbles-very careful interpretation is necessary in analysing any results.

Stroboscope technique can be improved with special lighting, for example where threads are used as an aid to visual observation, but such methods are unsuitable where there are gas bubbles in the flow.

The rotoscope has been designed in an attempt to overcome these limitations. It is similar to a Poggendorf mirror and comprises an electrically-driven prism rotating at half the speed of the impeller under investigation (see Fig. 1).

A pump impeller viewed through a rotoscope appears to be stationary. Relative particle paths and directions of motion can be shown up by taking a time exposure. By using stroboscopic lighting, they can be related to a time base and their velocity and acceleration defined.

This is a difficult instrument to set up. The optical axis of the prism must coincide with the axis of rotation. Several adjustment methods are available. Image stability depends on adjustment accuracy.

The speed of the pump tested varied from rest up to several thousand $\mathrm{r} . \mathrm{p}$. $\mathrm{m}$. The rotoscope faithfully followed the impeller motion and constantly gave a fixed image providing a fresh view of the various effects investigated and making them easier to comprehend. The equipment can also be used to study leakage along blades or at blade tips, or cavitation.

A large number of photographs of various techniques show such familiar effects as stream lines in various impeller types, swirl, bubble motion, and the flow pattern in a bladeless impeller.

Used in conjonction with such visual observation aids as threads or corpuscules, the rotoscope becomes an essential instrument for researchers and test bed operators interested in relative flows in hydraulic and gas flow rotary machines.

* Maître-Assistant à la chaire d’Electrotechnique, Faculté des Sciences de Paris. 